\title{
Méthode d'estimation rapide des volumes par catégories de grosseur en peuplements d'épicéa (Picea abies Karst)
}

\author{
J. RONDEUX (1), J. HEBERT (2), H. LECOMTE (2), A. TOUSSAINT (2) \\ Faculté des Sciences Agronomiques \\ Technologie forestière, Gembloux (Belgique)
}

\begin{abstract}
Résumé
Cette étude propose une méthode d'estimation rapide du volume à l'hectare total ou: ventilé par catégories de grosseur commerciales pour des peuplements d'épicéa (Picea abies KARST) situés dans la partie sud de la Belgique. L'analyse d'environ 1800 unités d'échantillonnage a permis d'établir des relations satisfaisantes entre les proportions de volume bois fort tige correspondant à diverses catégories de grosseur et la circonférence de l'arbre de surface terrière moyenne.
\end{abstract}

\section{Introduction}

L'estimation du volume à l'hectare des peuplements forestiers découle généralement de l'utilisation dinventaires complets ou d'inventaires relevant de telle ou telle méthode d'échantillonnage. Sur la base des données récoltées, le plus souvent des grosseurs à hauteur d'homme et des hauteurs moyennes d'arbres, des tarifs de cubage individuels adéquats permettent de déterminer des volumes correspondant à des limitations strictement définies (volume du bois fort de la tige, volume jusqu à une découpe en grosseur ou en longueur de tige, par exemple).

Dans le contexte de la gestion forestière à grande échelle, de l'appréciation rapide des ressources forestières ou de la valorisation des produits ligneux, il pent s'avérer très utile de disposer rapidement de chiffres relatifs au volume de l'ensemble du matériel sur pied ainsi qu'à sa ventilation par catégories de circonférence à $1.50 \mathrm{~m}$ du sol (3). Pour éviter de devoir procéder à de nombreux calculs portant a posteriori

(1) Technologie forestière.

(2) Programme de recherche "Inventaire des Ressources Forestières Wallonnes » financé par la Région Wallonne.

(3) Les catégories envisagées sont des catégories commerciales (usage belge) qui correspondent à : $0-39 \mathrm{~cm}, 40-69 \mathrm{~cm}, 70-89 \mathrm{~cm}, 90-119 \mathrm{~cm}, 120-149 \mathrm{~cm}, 150-179 \mathrm{~cm}, 180 \mathrm{~cm}$ et plus. 
sur les résultats fournis par un inventaire, nous avons étudié la possibilité d'estimer ces volumes par une méthode ne nécessitant quiun nombre très réduit de mesures ou de caractéristiques dendrométriques globales.

A cet effet, nous avons mis à profit la prodigieuse source d'informations que constituent plusieurs milliers d'unités d'échantillonnage installées et mesurées au sein de peuplements d'épicéa (Picea abies Karst.) à l'occasion d'un inventaire réalisé de 1980 à 1983 sur l'ensemble des forêts de Wallonie (Rondeux, 1983 a).

Nous envisagerons successivement la nature du matériel expérimental (paragraphe 2), la détermination du volume total à l'hectare des peuplements (paragraphe 3) et la manière de déterminer les volumes répartis par catégories de circonférence à $1,50 \mathrm{~m}$ (paragraphe 4). Les deux derniers paragraphes seront respectivement consacrés à l'utilisation pratique de la méthode proposée (paragraphe 5) et à diverses conclusions (paragraphe 6).

\section{Nature du matériel expérimental}

Les unités d'échantillonnage ou «placettes» qui ont servi de base à l'étude sont issues de pessières pures équiennes de tous âges réparties sur l'ensemble de la région wallonne. Près de 1800 unités dont la surface varie de 2 à 5 ares ont été analysées, elles sont représentatives de peuplements âgés de 20 à 110 ans, présentent des surfaces terrières moyennes à l'hectare échelonnées de $20 \mathrm{~m}^{2}$ à environ $50 \mathrm{~m}^{2}$ et des hauteurs dominantes situées entre $14 \mathrm{~m}$ et $36 \mathrm{~m}$. Les peuplements envisagés sont issus de tous les niveaux de productivité et sont représentés à raison de 9 p. 100 en classe 1, 32 p. 100 en classe 2,37 p. 100 en classe 3 et 22 p. 100 en classe 4 ; ces classes correspondent, par convention, à des hauteurs dominantes respectivement égales à $30 \mathrm{~m}, 27 \mathrm{~m}, 24 \mathrm{~m}$ et $21 \mathrm{~m}$ à 50 ans (DAGNelie et al., 1976).

On a pu vérifier qu'à l'intérieur de chacun de ces niveaux, l'évolution de la hautcur dominante en fonction de l'âge correspondait à celle définie dans la table de production établie pour l'épicéa commun en Ardenne méridionale (Thill \& Palm, 1976).

\section{Elaboration de fonctions de cubage}

Les données dendrométriques récoltées dans chaque placette avaient principalement trait aux circonférences à $1,5 \mathrm{~m}$ et aux hauteurs individuelles des arbres, elles ont été exploitées en vue de déterminer les caractéristiques globales ci-après :

- la circonférence de l'arbre de surface terrière moyenne ou circonférence quadratique moyenne,

1 - le volume du bois fort de la tige (4) et la surface terrière par placette et par hectare,

(4) Limité à la circonférence de $22 \mathrm{~cm}$ et calculé au moyen de tarifs de cubage individuels basés sur la circonférence à $1,5 \mathrm{~m}$ des arbres et sur la hauteur dominante des peuplements (PAlm, 1981). 
- le volume du bois fort de la tige réparti par catégories de circonférence à $1,5 \mathrm{~m}$ et par hectare,

- la hauteur dominante correspondant à la moyenne arithmétique des hauteurs des arbres les plus gros (à raison dun arbre par are de placette).

Nous référant à des considérations déjà émises lors d'études antérieures (Rondeux, 1977 ; Rondeux \& Toussaint, 1980), nous avons construit un tarif destiné au cubage direct des peuplements.

Ce tarif résulte d’un ajustement mathématique des volumes ramenés à l'hectare en fonction de la surface terrière à lhectare et de la hauteur dominante des peuplements; il se présente sous la forme suivante :

$$
\begin{aligned}
\mathrm{V}=-30,2814+1,1279 \mathrm{G}+1,5296 \text { Hdom }+ & 0,3922 \mathrm{G} . \text { Hdom } \\
& {[\mathrm{R}=0,9924] } \\
& {[\mathrm{CVR}=2,9 \text { p. } 100] }
\end{aligned}
$$

expression dans laquelle $\mathrm{V}$ représente le volume bois fort tige à l'hectare $\left(\mathrm{m}^{3}\right)$, $\mathrm{G}$ la surface terrière à l'hectare $\left(\mathrm{m}^{2}\right)$ et Hdom la hauteur dominante $(\mathrm{m})$.

Par la même occasion, nous avons également envisagé un modèle légèrement plus simple quant à son utilisation, soit :

$$
\begin{array}{ll}
\mathrm{V}=8,4650+0,4365 \text { G.Hdom } & \\
& {\left[\mathrm{R}^{2}=0,9921\right]} \\
& {[\mathrm{CVR}=3,0 \mathrm{p} \cdot 100]}
\end{array}
$$

Parcille méthode d'estimation du volume sur pied est particulièrement bien adaptée aux circonstances dans lesquelles rapidité et faible coût de réalisation sont préférés à un haut degré de précision (HAMILToN, 1975).

\section{Relations entre volumes globaux et volumes par catégories de grosseur}

Pour chacune des 1774 placettes analysées, nous avons déterminé les volumes des arbres relatifs aux différentes catégories de grosseur commerciales définies précédemment. Il s’est avéré que les rapports entre ces volumes et le volume de l'ensemble des arbres étaient étroitement liés à la circonférence quadratique moyenne et, quien outre, ils se caractérisaient par une assez grande stabilité, quels que soient les niveaux de productivité auxquels appartiennent les peuplements. Cela ne signifie pas pour autant que le niveau de productivité n'a pas d'effet sur la ventilation des volumes par catégories de grosseur. Il intervient de manière indirecte dans la mesure où il faut également tenir compte de l’âge des peuplements : si une circonférence moyenne donnée est la résultante des éclaircies pratiquées, elle est aussi en principe atteinte d'autant plus tôt dans la vie d'un peuplement que ce dernier est de productivité élevée.

Sur base de ces constatations, nous avons réparti les placettes inventoriées en classes de circonférence quadratique moyenne. Le volume de l'ensemble des arbres

(5) $\mathrm{R}^{2}$ et CVR reprósentent respectivement le coefficient de détermination et le coefficient de variation résiduelle. 
de chacune de ces classes a été ventilé par catégories de grosseur commerciales. Les volumes moyens ainsi obtenus, ramenés à l'hectare, ont été exprimés en pour cent du volume global et pondérés par les nombres de bois intervenant dans chaque placette en vue d'augmenter la précision de l'estimation des répartitions. L'évolution des pourcentages de volume en fonction de la circonférence quadratique moyenne des peuplements fait lobjet du tableau I. Ces pourcentages ne résultent pas dajustements mathématiques, ils correspondent à des valeurs moyennes.

Tableau 1

Pourcentages des volumes bois fort tige à l'hectare par catégories de circonférence à $1,5 \mathrm{~m}$, en fonction de la circonférence quadratique moyenne.

Percentages of volumes per hectare (to an upper girth of $22 \mathrm{~cm}$ ) by girth classes in relation with the quadratic mean girth of the stand.

\begin{tabular}{|c|c|c|c|c|c|c|c|}
\hline \multirow{2}{*}{$\begin{array}{l}\text { Circ. } \\
\text { moy. } \\
(\mathrm{cm})\end{array}$} & \multicolumn{6}{|c|}{ Catégories de circonférence $(\mathrm{cm})$ à $1,5 \mathrm{~m}$ du sol } & \multirow{2}{*}{$\begin{array}{c}\text { Nombre } \\
\text { plac. }\end{array}$} \\
\hline & -40 & $40-69$ & $70-89$ & $90-119$ & $120-149$ & 150 et + & \\
\hline 30 & 81,8 & 18,2 & 0,0 & 0.0 & 0,0 & 0,0 & 26 \\
\hline 35 & 47,6 & 52,1 & 0,3 & 0,0 & 0,0 & 0,0 & 89 \\
\hline 40 & 25,7 & 73,2 & 1,2 & 0,0 & 0,0 & 0,0 & 151 \\
\hline 45 & 13,9 & 82,5 & 3,7 & 0,0 & 0,0 & 0,0 & 126 \\
\hline 50 & 7,3 & 77,9 & 14,1 & 0,6 & 0,0 & 0,0 & 132 \\
\hline 55 & 4,3 & 67,7 & 26,1 & 1,8 & 0,0 & 0,0 & 117 \\
\hline 60 & 2,1 & 58,9 & 31,9 & 5,8 & 0,9 & 0,4 & 110 \\
\hline 65 & 1,0 & 46,6 & 41,4 & 11,0 & 0,0 & 0,0 & 86 \\
\hline 70 & 0,5 & 33,4 & 47,8 & 16,7 & $1, \mathbf{i}$ & 0,4 & 82 \\
\hline 75 & 0,3 & 21,7 & 50,7 & 26,8 & 0.5 & 0,0 & 101 \\
\hline 80 & 0,1 & 15,3 & 44,5 & 37,0 & 2,7 & 0,3 & 104 \\
\hline 85 & 0,1 & 9,4 & 40,3 & 44,6 & 5,5 & 0,0 & 86 \\
\hline 90 & 0,0 & 5,3 & 31,2 & 56,2 & 7,1 & 0,2 & 79 \\
\hline 95 & 0,0 & 3,4 & 21,9 & 62,8 & 11,7 & 0,2 & 85 \\
\hline 100 & 0,0 & 2,3 & 16,4 & 58,9 & 20,6 & 1,7 & 69 \\
\hline 105 & 0,0 & 1,3 & 11,6 & 56,7 & 27.2 & 3,2 & 63 \\
\hline 110 & 0,0 & 1,0 & 8,2 & 49,7 & 36.5 & 4,7 & 58 \\
\hline 115 & 0,0 & 0,8 & 4,9 & 42,1 & 42.3 & 9,9 & 53 \\
\hline 120 & 0,0 & 0,4 & 3,3 & 34,4 & 49,6 & 12,3 & 36 \\
\hline 125 & 0,0 & 0.2 & 2,4 & 26,5 & 53,7 & 17,2 & 36 \\
\hline 130 & 0,0 & 0,1 & 1,3 & 20,5 & 53,1 & 25,0 & 19 \\
\hline 135 & 0,0 & 0,1 & 1,0 & 16,6 & 47,3 & 35,1 & 24 \\
\hline 140 & 0,0 & 0,0 & 0,7 & 11,7 & 43,0 & 44,6 & 15 \\
\hline 145 & 0,0 & 0,1 & 0,3 & 9,5 & 38,7 & 51,5 & 11 \\
\hline 150 & 0,0 & 0,0 & 0,0 & 6,3 & 35.3 & 58,5 & 6 \\
\hline 155 & 0,0 & 0,1 & 0,2 & 5,2 & 25,4 & 69.0 & 10 \\
\hline
\end{tabular}

Le tableau 2 foumit, pour les 6 grandes catégories de circonférence à $1,50 \mathrm{~mm}$ envisagées, les différences en valeurs absolues entre les rapports moyens (pourcentages) calculés pour chaque classe de productivité $(1,2,3$ et 4 ) et les rapports calculés sur la totalité des placettes, abstraction faite des différences de productivité. Dans l'ensemble, il se confirme bien que les écarts sont peu importants si ce n'est dans certains cas particuliers intéressant la première classe de productivité, mais pour laquelle on notera aussi le nombre beaucoup moins élevé de placettes de référence. 


\section{TAileau 2}

Différences absolues entre les pourcentages de volumes calculés

pour chaque classe de productivité $[(1),(2),(3),(4)]$ et pour l'ensemble des classes en fonction des catégories de grosseur et de la circonférence quadratique moyenne du peuplement.

Absolute differences bitween volume percentages concerning the separate site classer $[(1),(2),(3),(4)]$ and all the sites in relation with girth classes and quadratic mean girth of the stand.

\begin{tabular}{|c|c|c|c|c|c|c|c|c|}
\hline \multirow{2}{*}{$\begin{array}{c}\text { Circ. } \\
\text { moy. } \\
\text { (cm) }\end{array}$} & \multicolumn{7}{|c|}{ Catégories de circonférnce $(\mathrm{cm})$ à $1,5 \mathrm{~m}$ du sol } & \multirow{2}{*}{$\begin{array}{c}\text { Nombre } \\
\text { plac. }\end{array}$} \\
\hline & & & $40-69$ & $70-89$ & $90-119$ & $120-149$ & $150 \mathrm{ct}+$ & \\
\hline 55 & $\begin{array}{l}\text { (1) } \\
(2) \\
(3) \\
(4)\end{array}$ & $\begin{array}{r}+0,2 \\
-0,7 \\
+0,7 \\
-0,1\end{array}$ & $\begin{array}{l}-0,8 \\
-0,2 \\
+1,6 \\
+1,3\end{array}$ & $\begin{array}{r}-\quad 0,9 \\
+\quad 0,3 \\
-\quad 1,0 \\
+\quad 0,7\end{array}$ & & & & $\begin{array}{r}5 \\
15 \\
26 \\
16\end{array}$ \\
\hline 65 & & & $\begin{array}{l}-4,6 \\
+1,5 \\
-1,1 \\
+2,5\end{array}$ & $\begin{array}{r}+\quad 0,6 \\
-\quad 1,6 \\
+\quad 2,4 \\
--3,3\end{array}$ & $\begin{array}{r}+3,8 \\
+0,5 \\
+1,3 \\
+0,5\end{array}$ & & & $\begin{array}{r}9 \\
17 \\
25 \\
18\end{array}$ \\
\hline 75 & & & $\begin{array}{c}+1,3 \\
- \\
+1,0 \\
-\end{array}$ & $\begin{array}{r}-\quad 6,5 \\
-\quad 5,0 \\
+\quad 3,0 \\
+\quad 5,0\end{array}$ & $\begin{array}{r}+6,0 \\
+5,0 \\
-3,9 \\
-4,4\end{array}$ & & & $\begin{array}{r}5 \\
31 \\
32 \\
17\end{array}$ \\
\hline 85 & & & $\begin{array}{l}+1,4 \\
+0,5 \\
-0,6 \\
+0,4\end{array}$ & $\begin{array}{r}-11,6 \\
-\quad 0,8 \\
+\quad 2,0 \\
+\quad 1,6\end{array}$ & $\begin{array}{l}+6,9 \\
+0,1 \\
-1,2 \\
+1,7\end{array}$ & $\begin{array}{l}+\quad 3,4 \\
+\quad 0,2 \\
-\quad 0,1 \\
-\quad 3,4\end{array}$ & & $\begin{array}{r}4 \\
28 \\
34 \\
13\end{array}$ \\
\hline 95 & & & $\begin{array}{c}-1,8 \\
+0,9 \\
-0,6 \\
-\end{array}$ & $\begin{array}{r}+\quad 6,7 \\
-\quad 1,6 \\
+\quad 0,7 \\
-\quad 0,4\end{array}$ & $\begin{array}{c}-3,8 \\
-0,1 \\
-1,2\end{array}$ & $\begin{array}{l}-\quad 0,8 \\
+\quad 0,9 \\
+\quad 0,1 \\
+\quad 0,6\end{array}$ & & $\begin{array}{r}5 \\
26 \\
30 \\
14\end{array}$ \\
\hline 105 & & & & $\begin{array}{r}+\quad 1,0 \\
+\quad 1,4 \\
-\quad 0,6 \\
-\quad 1,0\end{array}$ & $\begin{array}{r}-0,7 \\
-0,2 \\
+\quad 2,6 \\
-\end{array}$ & $\begin{array}{l}+\quad 0,8 \\
-\quad 0,1 \\
-\quad 1,6 \\
+\quad 1,9\end{array}$ & $\begin{array}{l}-\quad 0,8 \\
-\quad 0,7 \\
-\quad 0,5 \\
-\quad 0,9\end{array}$ & $\begin{array}{r}6 \\
14 \\
27 \\
12\end{array}$ \\
\hline 115 & & & & $\begin{aligned}- & 1,8 \\
+ & 1,0 \\
- & 0,4 \\
- & 0,2\end{aligned}$ & $\begin{array}{l}+5,4 \\
-4,0 \\
+3,9 \\
+2,3\end{array}$ & $\begin{array}{r}-0,1 \\
+\quad 3,4 \\
-\quad 4,9 \\
-\quad 2,3\end{array}$ & $\begin{array}{r}2,7 \\
-\quad 0,5 \\
+\quad 1,3 \\
+\quad 0,9\end{array}$ & $\begin{array}{r}4 \\
20 \\
17 \\
9\end{array}$ \\
\hline 125 & & & & $\begin{array}{l}-\quad 0,2 \\
-\quad 0,3 \\
+\quad 0,8 \\
-\quad 0,2\end{array}$ & $\begin{array}{l}+6,2 \\
-0,1 \\
-1,3 \\
+0,2\end{array}$ & $\begin{array}{r}-26,2 \\
+\quad 1,5 \\
+\quad 0,3 \\
+\quad 6,6\end{array}$ & $\begin{array}{r}+20,5 \\
-\quad 1,2 \\
+\quad 0,4 \\
-\quad 6,3\end{array}$ & $\begin{array}{r}2 \\
20 \\
10 \\
4\end{array}$ \\
\hline
\end{tabular}




\section{Utilisation de tables de conversion}

L'estimation de la répartition des volumes par catégories de grosseur commerciales suppose une détermination préalable de la circonférence quadratique moyenne et du volume total à lhectare.

Pour cette dernière variable, on peut faire usage du deuxième tarif de cubage peuplement évoqué (6) qui nécessite restimation de la hauteur dominante et de la surface terrière à thectare. Une autre manière de procéder, plus expéditive, mais aussi beaucoup plus approximative, est de recourir à une formulation moins courante du volume total à l'hectare, soit si le volume $V$ est exprimé en $m^{\prime \prime}$, la surface terrière $\mathrm{G}$ en $\mathrm{m}^{2}$ et la circonférence quadratique moyenne $c_{r}$ en $\mathrm{cm}$ :

$$
\mathrm{V} / \mathrm{G}=4,1810+0,07624 \mathrm{c}_{\mathrm{g}}
$$$$
\left[R^{2}=0,8714\right]
$$$$
[\mathrm{CVR}=8,7 \text { p. } 100]
$$

ou encore :

$$
V=\left(4,1810+0,07624 c_{x}\right) G,
$$

expression résultant de calculs ayant porté sur les données de base déjà traitées.

A titre d'exemple, si l'on considère un peuplement ou un massif dans lequel plusieurs points de sondage auraient fourni des estimations de la circonférence quadratique moyenne, de la surface terrière et de la hauteur dominante respectivement égales à $90 \mathrm{~cm}, 40,5 \mathrm{~m}^{2}$ et $25 \mathrm{~m}$, on peut déduire de l'équation [ $\mathrm{V}=8,4650+$ $0,4365 \mathrm{G} . \mathrm{Hdom}]$ et du tableau 1 les valeurs moyennes suivantes :

\begin{tabular}{c|c|c|c|c|c}
\hline \multirow{2}{*}{ Volume/ha } & \multicolumn{5}{|c}{ Volumes/ha et par catégories commerciales } \\
\cline { 2 - 5 } & $0-39$ & $40-69$ & $70-89$ & $90-119$ & $120-149$ \\
\hline \multirow{2}{*}{$450,4 \mathrm{~m}^{3}$} & $0 \%$ & $5,3 \%$ & $31,2 \%$ & $56,2 \%$ & $7,1 \%$ \\
& $0 \mathrm{~m}^{3}$ & $23,9 \mathrm{~m}^{3}$ & $140,5 \mathrm{~m}^{3}$ & $253,1 \mathrm{~m}^{3}$ & $32,0 \mathrm{~m}^{3}$ \\
\hline
\end{tabular}

Sur un plan pratique, cette procédure est relativement simple et rapide, mais il serait cependant plus rigoureux de calculer les volumes tant globaux que par catégories de circonférence à $1,50 \mathrm{~m}$, en chaque point de sondage et d'estimer ensuite les valeurs moyennes relatives à l'ensemble.

L'utilisation proprement dite de ces équations suppose évidemment la mise en auvre d'un échantillonnage adapté au degré d'homogénéité des peuplements et destiné à récolter les données de base : circonférence quadratique moyenne, hauteur dominante et surface terrière. Pour des peuplements d'épicéa suffisamment uniformes, on peut envisager de baser les estimations de ces caractéristiques sur environ 5 points de sondage, répartis autant que possible de manière systématique, pour des étendues

(6) Il est opportun d'attirer lattention sur le fait que ce tarif donne des résultats très voisins de ceux fournis par des tarifs du même type construits spécifiquement pour les zones. Nord (Rondeux, 1977) et Sud (Rondeux \& Toussaint, 1980) de localisation de l'épicéa commun en Belgique. 
allant jusqu'à 2 hectares, 8 points pour des étendues de 2 à 10 hectares, et 12 points au-delà de 10 hectares (Hamilton, 1975).

Dans le cas d'ensembles boisés plus importants comportant des peuplements d'âges différents, il conviendra de procéder à des échantillonnages spécifiques et d'augmenter le taux d'échantillonnage si la valiabilité des critères à estimer est plus ćlevée.

Sur un plan pratique, en chaque point sondé, la hauteur dominante résultera de la mesure des 2 plus gros arbres rencontrés dans un rayon de $8 \mathrm{~m}$, ou proche de celui-ci ; la surface terrière sera appréciée par l'intermédiaire d'un prisme relascopique ou du relascope de Bitterlich (RoNDEUx, 1983 b) tandis que la circonférence quadratique moyenne pourra être valablement estimée à partir des circonférences des 5 à 6 bois les plus proches du point de sondage (LAurent \& RondeuX, 1983).

\section{Conclusions}

La détermination rapide du volume à l'hectare d'un massif forestier ou d'un ensemble d'unités de gestion peut être réalisée à partir de tarifs de cubage établis à l'échelle des peuplements. En nous appuyant sur plusieurs milliers de mesures effectuées au sein de placettes d'échantilunnage installées en pessières pures équiennes et couvrant la totalité de la région wal onne, nous avons constaté que les rapports entre les volumes de différentes catégories de circonférence à $1,50 \mathrm{~m}$ et le volume global d'un peuplement pouvaient être sstimés, de manière satisfaisante, à partir de la circonférence quadratique moyenne.

La méthode proposée est intéressante à appliquer lorsqu'un peuplement d'épicéa est identifié par des caractéristiques dendrométriques globales telles que la surface terrière, la hauteur dominante et la circonférence quadratique moyenne. Elle ne présente évidemment plus le même intérêt si l'on dispose d'un inventaire détaillé du peuplement permettant de déterminer son volume par l'application d'un tarif de cubage individuel.

Elle offre enfin l'avantage d'être basée sur des critères simples permettant de couvrir un nombre élevé de situations aussi bien sur le plan de la productivité des. milieux que sur celui de la sylviculture pratiquée.

\section{Summary}

A way for rapid volume estimation by girth classes in spruce (Picea abies Karst.) stands

Results of large forest inventories have been analyzed in order to estimate timber product percentages in coniferous stands (Picea abies KARST) located in the southern part of Belgium.

Stand volume functions based upon top height and basal area give total volumes per hectare. The volumes estimated by commercial girth classes are presented as functions of mean stand girth. 


\section{Références bibliographiques}

Dagnelie P., Rondeux J., Thill A., 1976. Tables dendrométriques. Les Presses Agronomiques de Gembloux, $126 \mathrm{p}$.

Hamilton G.J., 1975. Forest mensuration handbook. Forestry Commission Booklet $n^{\circ} 39$, $274 \mathrm{p}$.

Laurent Ch., Rondeux J., 1983. Etude comparative de diverses unités d'échantillonnage à surfaces prédéterminées (Cas de forêts résineuses équiennes). Document 81.3. Centre de Recherche et de Promotion Forestières I.R.S.I.A. - Section "Aménagement et Production ». Faculté des Sciences Agronomiques, Gembloux, 21 p. $(+9$ annexes).

PALM R., 1981. Contribution méthodologique au cubage des arbres et à la construction de tables de cubage et d'assortiments. Thèse de doctorat. Faculté des Sciences Agronomiques de l'Etat, Gembloux, 295 p.

Rondeux J., 1977. Construction et utilisation de tarifs de cubage peuplement pour l'épicéa (Picea Abies KARST) en Ardenne méridionale. Bull. Rech. Agron. Gembloux, 12 (4), 339-348.

Rondeux J., Toussaint A., 1980. Tarif de cubage «peuplement 》 pour l'épicéa commun en Haute Ardenne septentrionale. Bull. Soc. Roy. For. Belgique, 87 (5), 227-231.

Rondrux J., 1983 a. Un inventaire forestier au service de la région wallonne. Wallonie 83, $10(1), 41-49$.

Rondeux J, 1983 b. La méthode de l'angle critique : une conception particulière de l'échantillonnage appliquée aux inventaires forestiers. Annales de Gembloux, 89, 183-200.

Thill A., Palm R., 1976. Production de l'épicéa commun dans le Sud-Ouest de l'Ardenne belge. Centre d'Ecologic Forestière I.R.S.I.A., Note technique $\mathrm{n}^{\circ} 28,42$ p. 